\title{
Revaluación del uso de algunas técnicas de la contabilidad de gestión
}

\author{
Diana Marcela Guerrero Ortiz ${ }^{1}$ \\ William Enrique Contreras Cuervo ${ }^{2}$
}

Recibido: 9 de febrero de 2014

Aprobado: 20 de mayo de 2014

\section{Guerrero, D.; Contreras, W. (2014) Revaluación del uso de algunas técnicas de la contabilidad de gestión. Activos 22, 137-152}

\section{Clasificación JEL: M49}

\section{Resumen}

El paso de la contabilidad de costos a la contabilidad de gestión fue un proceso de cambios que ocurrieron en un entorno altamente cambiante y que demandaba nuevos tipos de información para la gestión de las organizaciones. Además, ciertos parámetros que usan las organizaciones se ven alterados por los procesos de estandarización y globalización que se acercan rápidamente a nuestro entorno, obligando a que se diseñen ciertas medidas financieras enfocadas a unos usuarios específicos. El documento trata de responder a las siguientes preguntas: ¿cómo la contabilidad de gestión se ha venido introduciendo dentro del paradigma dominante en

1 Estudiante de Contaduría Pública de la Universidad Nacional de Colombia, integrantes del Grupo de Estudios en Contabilidad de Costos y Control de Gestión - Control + Ge de la Universidad Nacional de Colombia.

2 Estudiante de Contaduría Pública de la Universidad Nacional de Colombia, integrantes del Grupo de Estudios en Contabilidad de Costos y Control de Gestión - Control + Ge de la Universidad Nacional de Colombia. 
la contabilidad? ¿En qué forma ha venido desarrollándose para llegar a su estado actual? En este contexto se explora la aplicabilidad de algunos de los métodos propuestos por la contabilidad de gestión, por lo menos en lo que concierne a la teoría.

\title{
Palabras clave
}

Contabilidad de gestión, contabilidad de costos, usuarios, control, estandarización.

\section{Guerrero, D.; Contreras, W. (2014). Revaluation of the use of some techniques of management accounting. Activos 22, 137-152}

\begin{abstract}
The transition from costs accounting to management accounting was a process of changes which took place in a highly unstable environment and which required new types of information for organization management. Moreover, certain parameters used by organizations are altered by the globalization and standardization processes which are rapidly approaching our environment, forcing the design of specific financial measures focused on specific users. This document is trying to answer the following questions: How has management accounting been introduced into the dominant paradigms in accounting? How has it developed into its current state? In this context, the applicability of some of the methods proposed by management accounting is explored, at least in terms of theory.
\end{abstract}

\section{Keywords}

Management accounting, costs accounting, users, control, standardization. 
Guerrero, D.; Contreras, W. (2014). Réinvention de l'usage des quelques techniques de comptabilité de gestion. Activos 22, 137-152

\section{Résumé}

Lévolution de la comptabilité de budget à la comptabilité de gestion était un processus avec plusieurs de changements qui son déroulé dans un période avec divers changements qui demandaient nouveaux ressources d'information et gestion des entreprises. Aussi même quelque paramètres utilisés par les organisations sont altères par les nouveaux processus de standardisation et changements dues à la globalisation qui se reprochent de notre alentours, qui vont obliger à chercher nouvelles stratégies financières désignés pour usagers spécifiques. Dans ce document on va essayer de répondre aux questions suivantes comment la comptabilité de gestion a devenu aussi tôt important dedans le paradigme dominant de la comptabilité ? De quelle façon elle a-t-elle évolué jusquà nos jours ? Dans ce contexte on va explorer l'application des méthodes proposés par la comptabilité de gestion, au moins d'un façon théorique.

\section{Mots clés}

La comptabilité de management, la comptabilité de budget, les usagers, le contrôle, la standardisation. 
«Deberemos construir un horizonte futuro diferente para la contabilidad, buscando que su aporte no se centre solo en la prosperidad financiera y privada por parte de los agentes sociales» (Gómez, 2007).

\section{Introducción}

La contabilidad busca hoy en día cumplir con el objetivo de modelar los estados financieros dispuestos para efectuar las descripciones cualitativas necesarias ${ }^{3}$ sobre el estado económico de las organizaciones. El uso de sistemas de información cualificados, que garanticen la utilidad de la toma de decisiones tras un proceso de obtención, análisis y transmisión de información en formas altamente sofisticadas se vuelve un requerimiento fundamental en este proceso. Estos sistemas de información están en constante desarrollo, lo que implica que a lo largo de su evolución se presentan cambios que suelen manifestarse de distintas maneras: van desde la introducción de pequeñas mejoras en el cálculo de los costos o la presentación de informes, hasta la adopción de sistemas con indicadores (Alcarria y Martínez, 2003).

Los cambios surgidos en la legislación contable han provocado, a su vez, modificaciones en los comportamientos de los agentes que están relacionados con actividades inherentes a la profesión contable, concibiéndose así una respuesta a la constante necesidad de actualización, particularmente en lo que tiene que ver con la regulación.

La información juega un papel importante para los distintos actores inmersos en una organización, puesto que se debe ajustar a las necesidades de los usuarios, para establecer así la legitimidad del proceso que la contabilidad tiene como sistema de información que proporciona datos cuantitativos y cualitativos dentro del ámbito financiero y económico de las organizaciones; además, es el instrumento que tiene la capacidad de proveer el material necesario para conocer refinadamente las actividades

3 Según lo dispuesto tanto en la normatividad colombiana como en la reglamentación internacional, dependiendo el caso. 
que desarrolla la empresa. Existen entonces nuevos planteamientos, como Just in time, Kaizen costing y balance score card, los cuales proponen una contabilidad de gestión que permita un mayor control dentro de los aspectos de la empresa y no solo en términos contables.

Para explicar detalladamente cómo se ha llegado a este punto, se hará un recuento de cómo la contabilidad de gestión se ha adaptado a los cambios del entorno en el que ha tenido que desenvolverse desde sus inicios, para luego definir algunos de los nuevos planteamientos, y a partir de estos observar cómo se ha instaurado la nueva visión de la contabilidad de gestión como un instrumento imprescindible para la operación de las empresas.

\section{Desarrollo de la contabilidad de gestión}

Los historiadores de la contabilidad han recopilado evidencias sobre la importancia de los informes contables en diferentes culturas, y de cómo estos informes y registros han sido elaborados desde hace miles de años; incluso se han encontrado registros grabados en elementos tan simples como tablas de piedra (Johnson \& Kaplan, 1991). De igual forma, se han hallado evidencias de formas de contabilidad profesionalizadas y especializadas en culturas tan ancestrales como las de antiguo Egipto, o ejemplos de mecanismos avanzados de teneduría de libros en China, particularmente durante el reinado de la dinastía Chou (Hidalgo, 2005).

Sin embargo, aunque siempre ha existido la necesidad de llevar la contabilidad para conocer la economía de los individuos, el estudio de la contabilidad de gestión no tuvo los mismos inicios que la contabilidad en general. En este sentido, el nacimiento de la contabilidad de gestión es un fenómeno reciente que se puede ubicar hacia finales del siglo XIX y principios del XX (Johnson \& Kaplan, 1991). Los métodos usados en este tiempo no eran sofisticados porque las estimaciones de costos no tenían en cuenta los comportamientos de los productos ni las relaciones de los propietarios y empresarios con las demás personas que no formaban parte 
de la organización ${ }^{4}$. Aun con estas limitaciones, las manifestaciones de contabilidad de gestión satisfacían las necesidades de los usuarios.

Según Johnson y Kaplan (1991), los orígenes de la moderna contabilidad de gestión pueden hacerse coincidir con la emergencia de las empresas gestionadas jerárquicamente a comienzos del siglo XIX, como las armerías y las fábricas textiles. En este sentido, se puede afirmar que la contabilidad de gestión es un sistema pionero para el control del uso de las materias primas, que proporcionó de manera constante la información necesaria para distribuir con precisión responsabilidades y actividades necesarias dentro del proceso de producción de la compañía (Hidalgo, 2005).

Las medidas ofrecidas por la contabilidad de gestión al principio eran sencillas, pero servían adecuadamente a propietarios y gerentes, puesto que permitían que la gestión se concentrara en los costos primos y en el desarrollo de medidas de producción en forma de resumen, como el costo por hora o el costo por libra producida estimada tanto por procesos como por trabajador (Johnson \& Kaplan, 1991).

Al tiempo que se comenzaron a implementar los métodos de contabilidad de gestión en las nacientes empresas, aparecieron procesos productivos cada vez más complejos y elaborados en distintas etapas o procesos, de esta manera, la información acerca de la composición de costos en cada una de estas etapas se convirtió en una necesidad primordial. Se necesitó el uso de sistemas de información endógenos para ser usados en la toma de decisiones, lo que conllevó a aportar eficiencia a cada proceso productivo, permitiendo así el desarrollo de distintas conceptos, tales como costo por operación y razón de gastos operativos en relación con los ingresos (Hidalgo, 2005).

También se empezaron a usar medidas de margen bruto y ratio de rotación de stocks, en relación con las actividades de compra, almacenajey

4 Entendiendo como las personas que no formaban parte de la organización a los proveedores, clientes y demás agentes necesarios para realizar el objeto social de la compañía. 
venta, pues se buscaba medir la rentabilidad y rapidez con que las mercancías eran adquiridas y vendidas por la empresa (Johnson \& Kaplan, 1991).

En el periodo comprendido entre 1885 y 1900, los sistemas de contabilidad de gestión se centraron en los costos que se podían medir fácilmente, especialmente en lo que se refería a los materiales y la mano de obra. Pero el inconveniente de los procesos de medición se presentaba en el momento de medir los costos indirectos de fabricación, por lo que fue necesario establecer criterios para aquellos productos poco homogéneos o que no se podían incorporar de manera directa al proceso de costeo. Esto marcó el inicio de los métodos que proponen asignar los costos indirectos de fabricación a partir del uso de mano de obra directa.

En 1903, la DuPont Company ${ }^{5}$ inició el diseño de un sistema de operación y presupuesto capaz de coordinar las actividades y asignar recursos a sus grupos operativos (Johnson \& Kaplan, 1991). Sin embargo, la innovación más importante en materia de contabilidad de gestión fue la medida del retorno sobre la inversión (return on investment, $R O I^{6}$ ); fue así como los directivos usaron el ROI para orientar la asignación de capital a las divisiones, garantizando unos criterios de priorización de recursos hacia aquellas que resultaban más rentables Hidalgo (2005).

El ROI se extendió como indicador fundamental en las primeras décadas del siglo XX, justo cuando aparecieron las empresas multidivisionales, en las cuales era necesario que se gestionara desde un único centro de decisión. Esto implicaba que la operación y el proceso productivo deberían hacerse en el interior de cada división, por lo que era necesaria la distribución de responsabilidades entre las distintas divisiones, de modo que se asegurara que la marcha de cada división estuviera acorde con los objetivos de la empresa (Johnson \& Kaplan, 1991).

5 Compañía constituida en 1903 a partir de una unión de empresas familiares independientes, dando lugar a una forma corporativa nueva e interesante desde la gestión.

6 Indicador que proporciona una medida global del éxito de cada inversión. 
En este contexto, según Hidalgo (2005, p. 98), surgió un conjunto de nuevas funciones básicas de la contabilidad de costos:

a) Ayudar a determinar el precio satisfactorio de los bienes vendidos.

b) Ayudar a fijar un límite mínimo en las disminuciones de precios.

c) Determinar qué productos eran lo más beneficiosos.

d) Controlar el inventario.

e) Fijar un valor para el inventario.

f) Comprobar la eficacia y eficiencia de los distintos departamentos y procesos.

g) Detectar pérdidas, derroches y robos de inventarios.

h) Separar los costos de la inactividad de los costes de producir bienes.

i) Ayudar en los informes financieros.

Hasta entonces, las prácticas de la contabilidad de gestión habían evolucionado, siendo cada vez más diversas y complejas, de tal forma que pudieron satisfacer las necesidades de información y control de los directivos de las organizaciones. No obstante, en ese momento el ritmo de la innovación en cuanto a la medición y valoración de costos pareció detenerse; la razón de este estancamiento se explica por el auge del análisis de los estados contables financieros gracias a la ampliación y profundización de los mercados de capitales, la mayor demanda sobre la valoración de inventarios y la creciente necesidad que tenían las crecientes estructuras corporativas de capturar fondos del público (Johnson \& Kaplan, 1991; Hidalgo, 2005).

Como consecuencia de ese estancamiento del desarrollo de los sistemas de contabilidad de gestión, durante años se abandonó la prioridad de la 
producción de información oportuna y detallada sobre la eficiencia del proceso productivo; tampoco se logró centrar la atención en la gestión de los insumos productivos (básicamente materiales y mano de obra directa). Esta situación condujo a que las compañías tuvieran información distorsionada sobre estos elementos y a que se generara un contexto favorable para la toma errada de decisiones en cuanto a la determinación de costos, e incluso a tener una idea equivocada sobre el impacto de un producto tanto en el proceso de producción como en el mercado.

Años después la contabilidad de gestión vivió un nuevo auge, en el que se desarrollaron tres etapas importantes que respondían a la evolución de sus objetivos. Estas son las que se conocen en la literatura como 1) etapa del coste verdadero, 2) etapa de la verdad condicionada y 3 ) etapa de la verdad costosa (Horngren, 1991).

La etapa del coste verdadero comenzó en los años 40 del siglo XX y se extiendió hasta 1960. Se caracterizó por la búsqueda de la exactitud en los cálculos de los costos. Concebía la minuciosidad en el análisis del coste y la utilización de normas rigurosas de medida como método para determinar el coste completo con base en el coste total o full cost (Ortega, 1999).

La etapa de la verdad condicionada inició durante la década de los años 60 del siglo XX. Surgió de una crítica al modelo de exactitud de costos, a partir de la cual planteó la necesidad de buscar la mejor información para cada receptor o usuario. Esta fue la etapa de surgimiento del método del costeo variable (direct costing), y se incorporó en procesos de toma de decisiones internas y de control. Este modelo se vio favorecido por los avances en los campos de la investigación operativa, las matemáticas aplicadas a la economía y la estadística (Hidalgo, 2005).

Finalmente, la etapa de la verdad costosa tuvo lugar a finales de los años 70 del pasado siglo. Esta etapa consideró los sistemas de contabilidad de gestión como parte de los sistemas de información generales de la empresa, pues aportaba datos e información a todas las estructuras de la organización y no se limitaba al cálculo de costos (Ortega, 1999). En 
este punto se hizo necesaria la incorporación de tecnologías para hacer una implementación de sistemas de información capaces de suministrar continuos informes sobre la situación del trabajo y para controlar procesos de manera más eficaz.

Sumado a esto, la revolución computacional que se llevó a cabo en esta época apoyó la reducción de los costos de información y recolección de datos, además de permitir que dichos sistemas fueran asequibles aun para organizaciones pequeñas, lo que contribuyó a la mejora de los procesos de planificación y control, hasta tal punto que el coste y el valor de la información se equilibrasen en un punto óptimo (Hidalgo, 2005).

En este sentido, autores como Kaplan (1984) indican que los cambios del entorno competitivo de los años 80 hicieron reexaminar la contabilidad de costos tradicional y los sistemas de control de gestión, pues hubo un cambio en la naturaleza de las organizaciones y de la dimensión de la competencia. En la década de los 80 , las empresa redescubrieron el papel fundamental que jugaba la ventaja competitiva y se comprometieron a mejorar la calidad de los procesos productivos y del diseño de los productos, así como también la disminución de los niveles de inventarios y el tiempo de proceso. Así mismo, se empezó a considerar el sistema de fabricación de justo a tiempo en la producción y la distribución.

Por otro lado, el entorno de producción se modificó ${ }^{7}$, y tal como señala Hidalgo (2005, p. 87), esto se caracterizó por:

(I) Cambios rápidos en la tecnología, que hicieron que aumentase la productividad de forma exponencial; (II) la aparición de productos con un ciclo de vida corto, que hizo a las empresas lanzar al mercado continuamente productos casi perecederos; (III) cambios en la organización

7 Como consecuencia de la irrupción de los productos japoneses en los mercados occidentales. 
de la producción, pasando de una organización por funciones ${ }^{8}$ a una producción integrada?.

Este nuevo entorno económico consideraba desde la expansión de las tecnologías de la información y la competencia global hasta las innovaciones tecnológicas y organizacionales, y tuvo impactos considerables en los procesos de fijación de objetivos a corto y largo plazo, en las formas de medir la eficiencia y rentabilidad de los productos y, lo más importante, sobre la toma de decisiones en función de las nuevas necesidades del entorno (Hidalgo, 2005).

Johnson y Kaplan (1991) afirman, por tanto, que la contabilidad de gestión es un componente indispensable en la estrategia de la empresa para alcanzar el éxito competitivo. Sin embargo, un sistema de gestión excelente no garantiza el éxito, sino que además se hace necesario disponer de métodos de recolección, procesamiento y disposición de información de manera sofisticada, así como también es necesario ofrecer los productos que los clientes esperan y en los que se orienten sistemas de producción y distribución altamente eficientes.

Finalmente, estos cambios llevaron a la aparición de nuevos modelos de contabilidad de gestión durante la década de los 90, muchos de ellos vigentes actualmente: los sistemas de costes por actividad ( $\mathrm{ABC}$ ), el costeo Kaizen, el desarrollo de los planteamientos de control de calidad total (TQM), los sistemas de planificación de las necesidades de los materiales (MRP), las tecnologías de producción óptima (OPT), las técnicas de justo a tiempo (JIT) y los sistemas de fabricación flexible (FMS). De manera más reciente aparecieron en el contexto la contabilidad de gestión medio ambiental, los indicadores de gestión integrales (BSC) y la utilización de sistemas expertos (Hidalgo, 2005).

8 En la que el producto pasa por una serie de fases.

9 En la que el producto se comenzaba y terminaba en un mismo proceso. 
Paulatinamente, estos modelos fueron consolidando un conjunto de cuatro objetivos fundamentales que todo sistema de contabilidad de gestión debe cumplir en atención de ofrecer información para el control de los planes de la empresa. Estos objetivos están plasmados en la figura 1.

Figura 1. Objetivos básicos de los sistemas de contabilidad de gestión

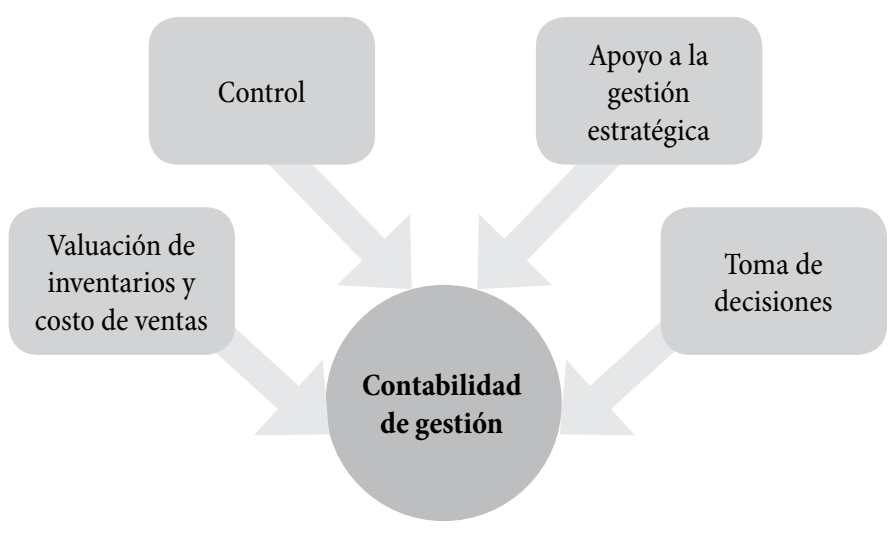

Fuente: elaboración propia.

A partir de dichos objetivos se crearon nuevos métodos de control que permitieron a las empresas ajustarse a las nuevas necesidades del entorno. Los más importantes, sin duda, debido a su utilización y a la calidad de información que proporcionaron para la gestión, son el Kaizen costing, el just in time y el cuadro de mando integral o balanced score card, instrumentos que señalaron el camino de su evolución y el aumento de sus niveles. A continuación se explica el desarrollo de cada uno.

El Kaizen costing es una técnica de control muy efectiva. Desde sus inicios en el Japón de la Segunda Posguerra Mundial, esta técnica contribuyó al mejoramiento notorio de la calidad de sus productos, con los que no podían competir dentro del mercado internacional. Varios autores atribuyen el posicionamiento japonés en lo más alto de los mercados mundiales como resultado de la aplicación de este instrumento y 
el consecuente perfeccionamiento de la calidad y el diseño de productos (America, Caribbean, \& Service, 1995). El costeo Kaizen le permite a las empresas desenvolverse de mejor manera en el entorno económico actual, determinado por la globalización y los rápidos cambios culturales y tecnológicos, a la vez que proporciona mecanismos para superar problemas tales como la contaminación ambiental y las consecuencias derivadas de la sobreexplotación de los recursos escasos. Es en este punto donde las empresas se ven obligadas a hacer más efectivas sus etapas productivas y en el que aprenden a usar eficazmente los recursos con los que cuentan.

El just in time o justo a tiempo (JIT) se considera como una puntualización de la filosofía de gestión japonesa aplicada al proceso productivo, la cual implica tener elementos de calidad y cantidad en el lugar correcto y en el momento adecuado. Por medio de la implementación del JIT, se busca el aumento de la calidad, la productividad y la eficiencia, así como el mejoramiento de la comunicación intraorganizacional y la disminución de costos y desechos (Cheng, Podolsky, \& Jarvis, 1996). Y aunque la técnica de JIT tiene una amplia tradición, su adaptación en la empresa moderna es relativamente nueva, pues hace necesario desarrollar nuevos enfoques en respuesta a entornos cada vez más competitivos.

Finalmente, el cuadro de mando integral o balanced score card es un instrumento estratégico útil para las empresas, que a diferencia de los métodos tradicionales es compatible con sistemas de control que gira alrededor de indicadores y medidas tanto financieras como no financieras. El balanced score card además exige una estrecha relación entre las acciones de hoy y las metas futuras, lo que permite la traducción de objetivos estratégicos en resultados, centrando la atención del staff directivo en la consecución o logro de los objetivos estratégicos pronosticados. En otras palabras, el cuadro de mando integral es un sistema de comunicación, información y formación (Muñiz \& Monfort, 2005, p. 30).

Con estos métodos, las empresas buscan abrirse campo en el panorama de la competitividad global; su aplicación permite a las compañías minimizar costos y tiempos de producción, aumentar la productividad y mejorar la 
calidad de cada uno de sus productos, permitiendo de esta forma aumentar los campos de acción de la compañía.

Sin embargo, estos métodos se enfocan únicamente en la empresa y en la capacidad para generar buenos resultados financieros, dejando de lado al trabajador, el cual se analiza simplemente como fuerza de trabajo y mano de obra, no como persona; esto les permite a las empresas otorgar y generar sistemas y unidades de medida sobre el trabajador a partir de su capacidad de producción y generación de resultados. En casos como el de la industria de producción de calzado, en el que se paga por productos de excelente calidad, elaborados en cortos plazos de tiempo, en los que un trabajador normal no alcanzaría a llevar a cabo la totalidad del producto, generan a su vez problemas de salud en sus trabajadores y ponen en riesgo tanto la capacidad de producción de la empresa como la integridad de sus empleados.

En este sentido es necesario resaltar lo siguiente:

El elemento humano en la producción, y más importante, en cada persona, puede ahora reconocerse a través de sus contribuciones a la eficiencia de la empresa [...] el intento de la administración científica por adueñarse de la vida laboral del individuo con el fin de intervenirla y optimizar su eficiencia. (Miller, 1987, p. 55)

\section{Resultados y conclusiones}

El presente trabajo plantea que la implantación y el uso que se le da a la contabilidad de gestión tienen unos fines de estandarización, y que su aplicación dentro de las empresas está siendo utilizada como medida de desempeño, calidad del producto y de la productividad. De esta manera:

De ser un instrumento de carácter exclusivamente jurídico y con una clara vocación de registro, la contabilidad ha pasado a ser una disciplina que 
proporciona no solamente información financiera, sino también información de contenido social destinada a la colectividad. (Archel, 2001, p. 34)

Este cambio genera beneficios para la empresa y un autocontrol por parte de los gerentes y su equipo de trabajo, puesto que la empresa produce planes de presupuestos, objetivos a corto y largo plazo a los que los empleados deberán ceñirse, provocando que se geste una autorregulación evaluada por el desempeño de todo el personal.

Finalmente, el hecho de que la información ofrecida por la contabilidad esté en función de las necesidades de los agentes interesados recalca la relevancia que deben tener las decisiones que se tomen, pues se busca que la empresa reaccione de la manera en que la sociedad espera que lo haga. Es decir, se legitima aún más el valor de participación que tiene la contabilidad cuando se logra que el sistema de valores de una empresa sea congruente con el sistema de valores de la sociedad. En efecto, las organizaciones han optado por tener políticas de información que estén guiadas por la necesidad de justificar ante la sociedad determinadas actuaciones empresariales (Etkin, 2005).

Y lograr esto es una tarea fácil, puesto que la contabilidad de gestión no se encuentra estrictamente regulada por medio de un marco legislativo que le permita recopilar, almacenar, analizar, evaluar y presentar información relacionada con el proceso productivo de una compañía, ya que existen diferentes posibilidades de aplicarla. De hecho, se busca que la contabilidad de gestión funcione como un sistema de información que proporcione datos a los diferentes usuarios, facilitando el proceso de toma de decisiones. 


\section{Referencias bibliográficas}

Alcarria, J. y Y. Martínez, M. (2003). Prácticas y usos de contabilidad de gestión. Partida doble 144, 90-103.

Archel, P. (2001). Algunos determinantes de la información medio ambiental divulgada por las empresas españolas cotizadas. Revista de Contabilidad, 4(7), 129-153.

CEPAL. (1995). Notas sobre la economía y el desarrollo.

Cheng, T. C. E., Podolsky, S., \& Jarvis, P. (1996). Just-in-time manufacturing: an

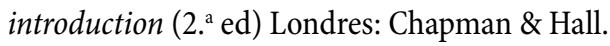

Etkin, J. (2005). Gestión de la complejidad en las organizaciones. México: Ediciones Granica.

Gómez, M. (2007). Pensamiento único y contabilidad: la posibilidad de la contaminación como disciplina moral. Revista Facultad de Ciencias Económicas: Investigación y Reflexión, (15)2, 29-43.

Hidalgo, F. G. (2005). Evolución histórica de la contabilidad de costes y gestión. Revista Española de Historia de la Contabilidad, 2, 100-122.

Horngren, C. T. (1991). Contabilidad de costos: un enfoque gerencial. México: Pearson Educación.

Johnson, H. T. \& Kaplan, R. S. (1991). Relevance lost: the rise and fall of management accounting. Boston: Harvard Business School Press.

Kaplan, R. S. (1984). The evolution of management accounting. The Accounting Review, 3, 390-418.

Miller, P. O. L. T. (1987/2009). La contabilidad y la construcción de la persona gobernable. En M. Gómez y C. Ospina (Ed.) (2009), Avances interdisciplinarios para una comprensión crítica de la contabilidad. Textos paradigmáticos de las corrientes heterodoxas. Bogotá: Escuela de Administración y Contaduría Pública, Universidad Nacional de Colombia y Departamento de Ciencias Contables de la Universidad de Antioquia.

Muñiz, L. \& Monfort, E. (2005). Aplicación práctica del cuadro de mando integral. Barcelona: Ediciones Gestión 2000.

Ortega, J. A. (1999). Introducción a la contabilidad de gestión. Comillas: Universidad Pontificia Comillas. 